\title{
Immediate Implant Placement and Provisionalization: Optimizing Esthetics and Function
}

\author{
${ }^{1}$ Mohammed Fayaz Pasha, ${ }^{2}$ Soumyadev Satpathy, ${ }^{3}$ Shilpa Shetty, ${ }^{4}$ Rohit Pandurangappa, ${ }^{5}$ Sana Taher
}

\begin{abstract}
Esthetic replacement of tooth in the anterior maxilla is one of the most critical demands of any patient seeking tooth replacement. One of the major current challenges is oral rehabilitation after tooth loss, due to fracture. Esthetic and functional results, together with periodontal health, predict success and prognosis. Implant dentistry provides the solutions for the placement of osseointegrated screw-retained implants or cemented prostheses to replace missing teeth. Careful use of provisional prostheses preserves the harmonious architecture of gingival tissues and affects final treatment results positively. This study describes series of three clinical cases of root fracture of maxillary anterior teeth, which had been previously endodontically treated. The teeth were extracted, immediate implants were placed and the provisional prostheses were fabricated and immediately loaded. The use of an immediate provisional restoration is a viable technique that has good esthetic and functional results and preserves gingival architecture and provides excellent esthetic result for the patient.
\end{abstract}

Keywords: Implant, Immediate implant placement, Immediate loading, Esthetics, Immediate provisional restoration.

How to cite this article: Pasha MF, Satpathy S, Shetty S, Pandurangappa R, Taher S. Immediate Implant Placement and Provisionalization: Optimizing Esthetics and Function. Int J Oral Implantol Clin Res 2014;5(2):72-78.

Source of support: Nil

Conflict of interest: None

\section{INTRODUCTION}

Choosing the most appropriate protocol for the rehabilitation of the partially edentulous jaw may represent a challenge and the clinician should rely on evidence based, thorough information. The edentulous predicament is directly related to alteration of facial esthetics as well as loss of ability to chew, taste and smile. Treatment of the partially edentulous jaw with dental implants represents a scientifically and clinically validated treatment modality. ${ }^{1,2}$

\footnotetext{
${ }^{1,4}$ Reader, ${ }^{2,5}$ Postgraduate Student, ${ }^{3}$ Professor and Head

${ }^{1-4}$ Department of Prosthodontics and Implantology, VS Dental College and Hospital, Bengaluru, Karnataka, India

${ }^{5}$ Department of Orthodontics, VS Dental College and Hospital Bengaluru, Karnataka, India
}

Corresponding Author: Soumyadev Satpathy, Postgraduate Student, Department of Prosthodontics and Implantology VS Dental College and Hospital, Bengaluru, Karnataka, India Phone: 9036755475, e-mail: soumyadevster@gmail.com
The extended healing time without implant loading associated with the conventional loading protocol is a disadvantage from the patient's perspective. Hence, reducing the time to loading, would be of great benefit to the patient. Today, many implant surgical and prosthetic concepts are used for the treatment of the edentulous jaw. Rough implant surfaces and immediate or early loading protocols have led to faster healing times and immediate or early restoration of function and esthetics in carefully selected cases. ${ }^{3}$

The different times for loading dental implants have been somewhat debatable in the past; however, in accordance with recently published reports, the following current definitions were used for the present case report: ${ }^{4}$

- Immediate loading: A prosthesis is connected to the dental implants within 1 week following implant placement.

- Early loading: A prosthesis is connected to the dental implants between 1 week and 2 months following implant placement.

- Conventional loading: Dental implants are allowed to heal for a period greater than 3 months after implant placement without connection of a prosthesis in mandible and 6 months in the maxilla.

Hence, the purpose of this case report is to demonstrate the technique of immediate loading with immediate provisionalization, on immediately placed implant following extraction.

This case series describes three cases of root fracture of endodontically treated maxillary anteriors, which were treated with extraction of the affected tooth followed by immediate implant placement and provisional prostheses, were placed immediately.

\section{GOAL}

This case series describes three cases of immediate implant placement and delivery of a fixed transitional restoration on the same day. It explores problems and concerns related to this treatment concept.

\section{CASE REPORTS}

\section{Case 1}

A 36-year-old female presented to Department of Implantology of VS Dental College and Hospital, Bengaluru, Karnataka, India, with a chief complaint of fractured 
upper right central incisor (tooth \#8) (Fig. 1). Patient gave a history of root canal treatment done 6 months back, following which patient had undergone fixed dental prosthesis with respect to the same.

Clinical examination revealed signs of mobility of the fractured crown portion of the tooth. The radiographic picture showed a horizontal radiolucent line below the alveolar crest indicating fracture line (Figs 2 and 3). The patient was diagnosed with a root fracture at the junction of middle and cervical third.

The treatment plan consisted of extraction of the tooth and immediate implant placement. The patient was evaluated for local and systemic factors. Since, it was a immediate implantation procedure, the cortical plate was critically evaluated by means of periodontal probe to check for continuity.

The tooth was extracted with an atraumatic technique and a periotome was used to preserve the osseous structures surrounding the socket (Figs 4 and 5). The extracted tooth was measured at the level of the cervical portion of the root and also the length of the root was measured (Figs 6 and 7). The crestal diameter was $4.5 \mathrm{~mm}$. Then, an osteotomy was performed, beyond the apex of the socket, to a depth of

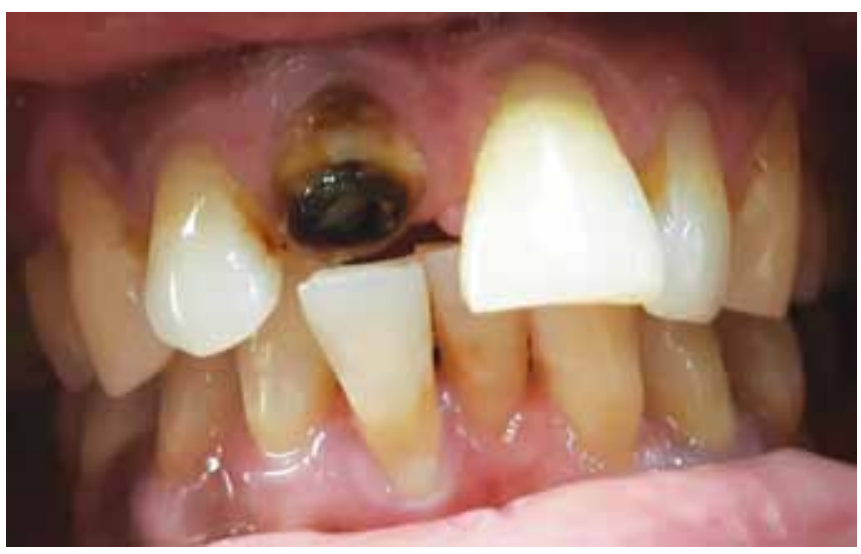

Fig. 1: Preoperative view of the fractured upper right central incisor

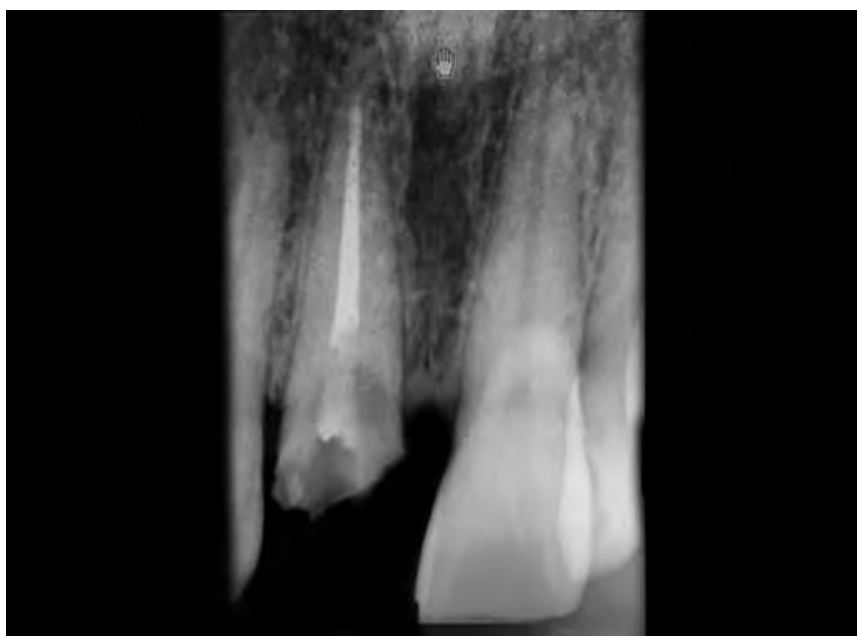

Fig. 3: Radiographic view showing the horizontal fracture line
$5 \mathrm{~mm}$ using the alveolar crest as a landmark and following a slightly more palatal direction. The integrity of the socket walls was verified with the use of a periodontal probe. A root-form implant of $5 \times 13 \mathrm{~mm}$ was inserted (Figs 8 to 10). The implant shoulder was located $3 \mathrm{~mm}$ apical to the cementoenamel junction of the adjacent left central incisor. The distance between adjacent teeth and implant was $3 \mathrm{~mm}$. No graft and/or membranes were used as there was no jumping distance between the implant external surface and prepared bone within the osteotomy. A primary stability of $35 \mathrm{Ncm}^{2}$ was obtained.

The standard pre-fabricated abutment as supplied by the manufacturer of the used implant system was screwed in to the implant and was checked for fit and stability. Following which, the abutment was modified to compensate for the labial prominence to get a $15^{\circ}$ angulation (Figs 11 and 12).

To obtain the best esthetic and functional results possible, as well as to respond to the patient's expectations of having the provisional match her natural teeth, a careful local and general risk analysis was conducted, and plans were made to use a modified acrylic tooth, as the provisional prosthesis for implant loading.

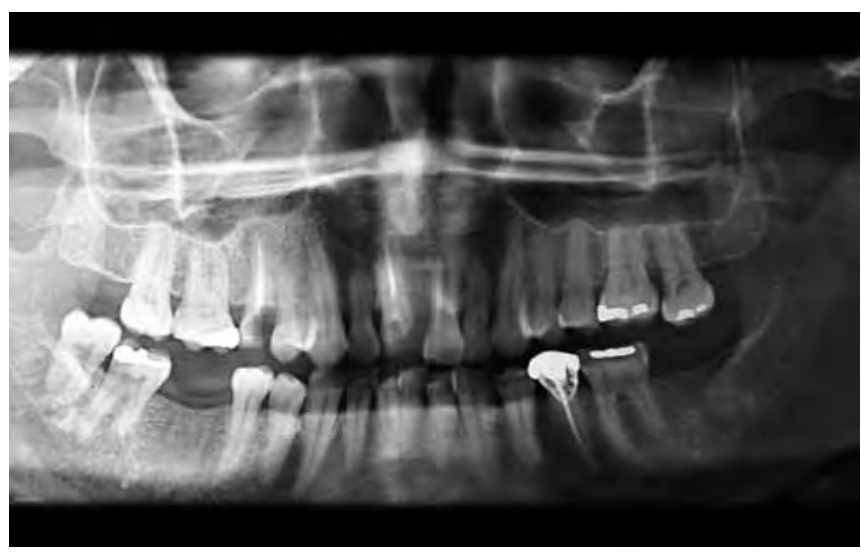

Fig. 2: Orthopantomograph showing the fractured tooth

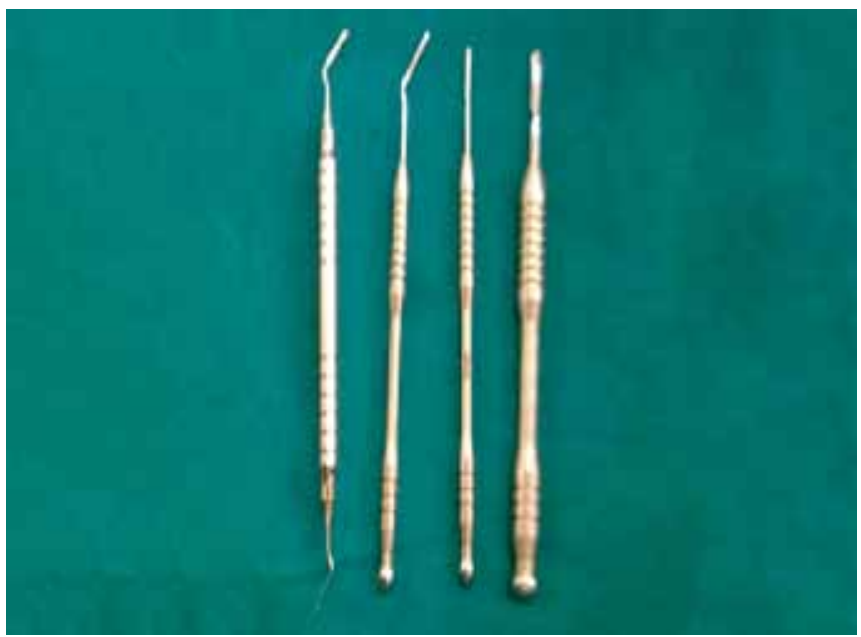

Fig. 4: Instruments used to extract the tooth 


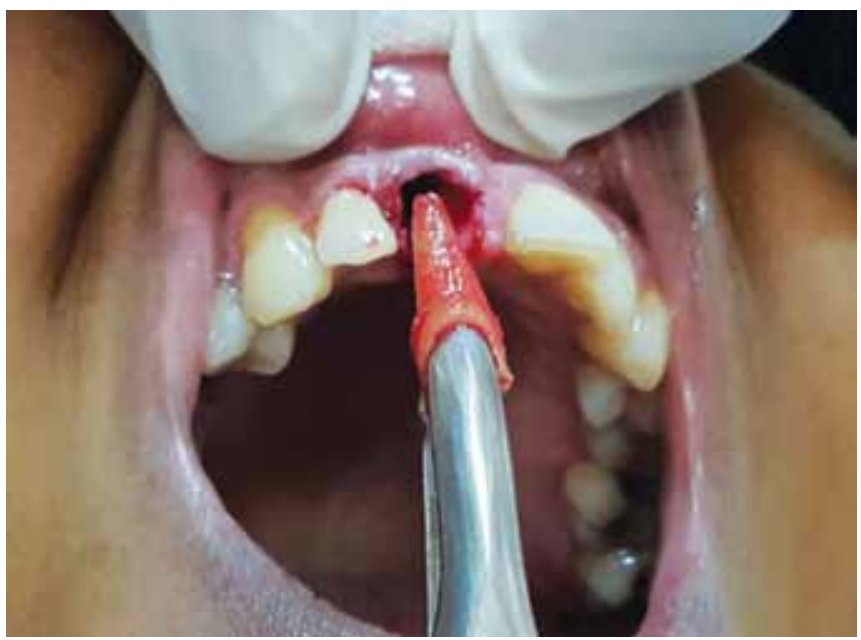

Fig. 5: Tooth extracted atraumatically

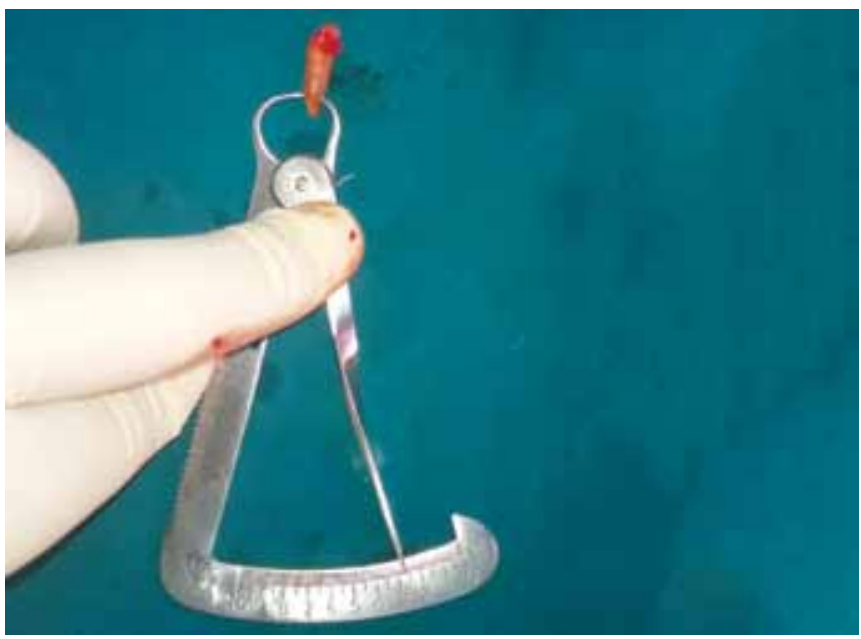

Fig. 7: Using a gauge, the diameter at the apex of extracted tooth root is measured

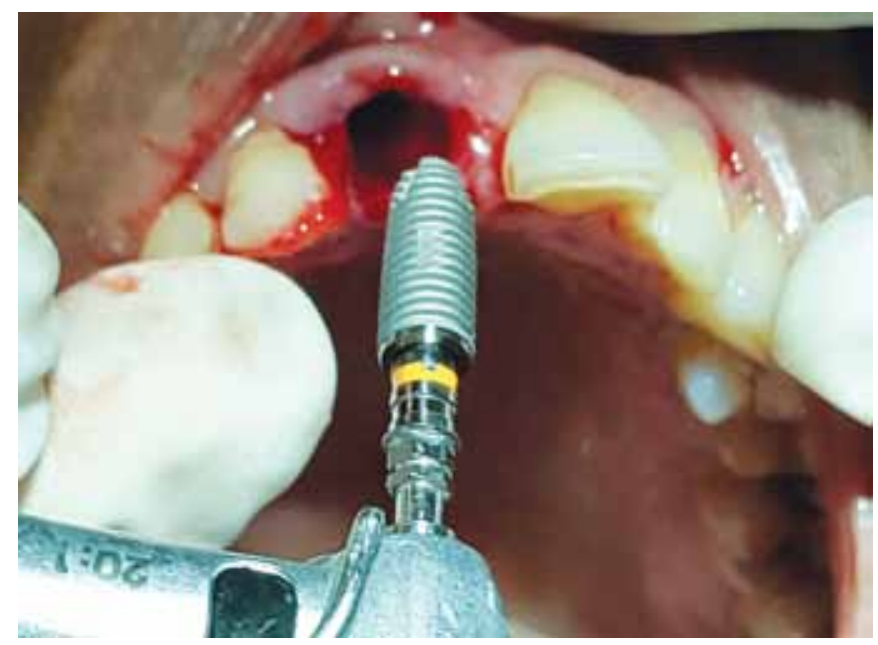

Fig. 9: Implant being placed

To fabricate the implant-supported provisional restoration, the acrylic tooth was prepared into a facet, which was then rebased over the prepared abutment using a light cured composite resin. The quality of the polishing of light cured composite resin surfaces, when compared with that

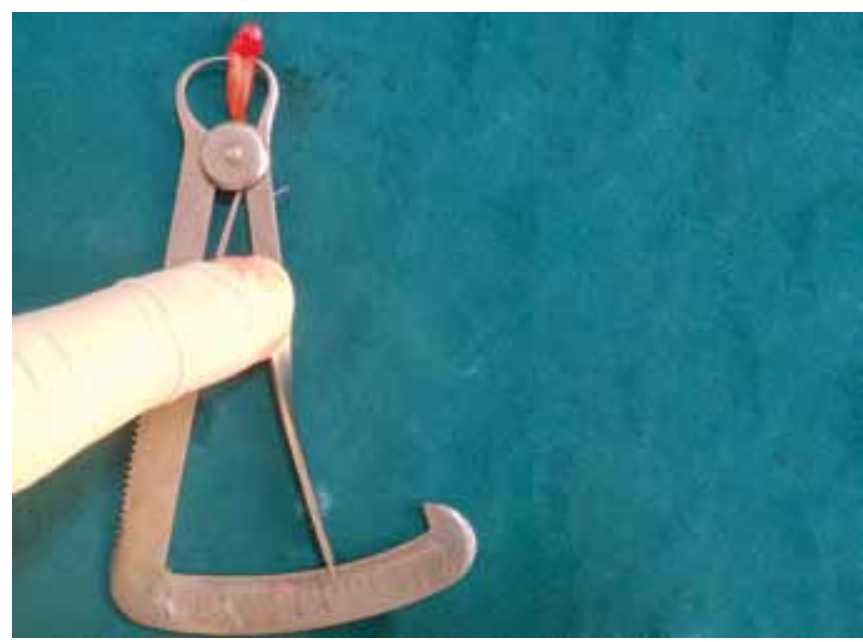

Fig. 6: Using a gauge, the diameter at the coronal portion of extracted tooth root is measured

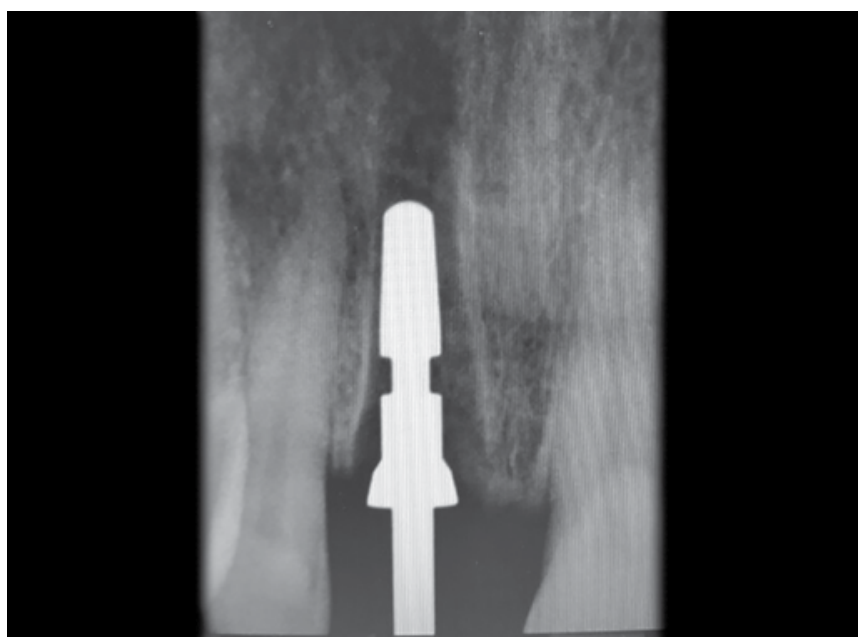

Fig. 8: Paralleling pin placed to show the location of the implant

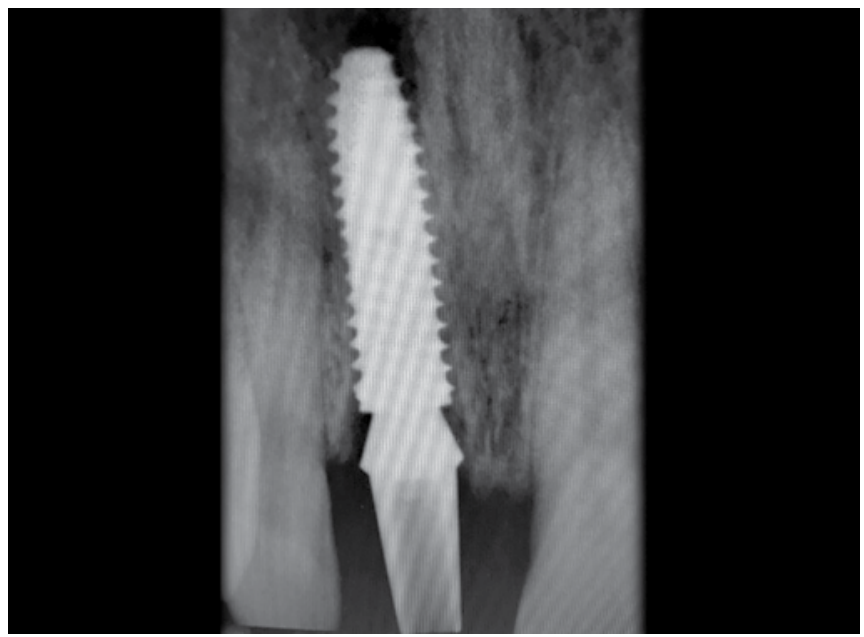

Fig. 10: Radiograph showing implant placed in the osteotomy site

of acrylic resins, makes cleaning easier and, consequently, promotes gingival health in the region (Figs 13 and 14).

However, the cervical contour of the provisional is responsible for tissue stability at the gingiva-tooth-implant interface. $^{11,12}$ 


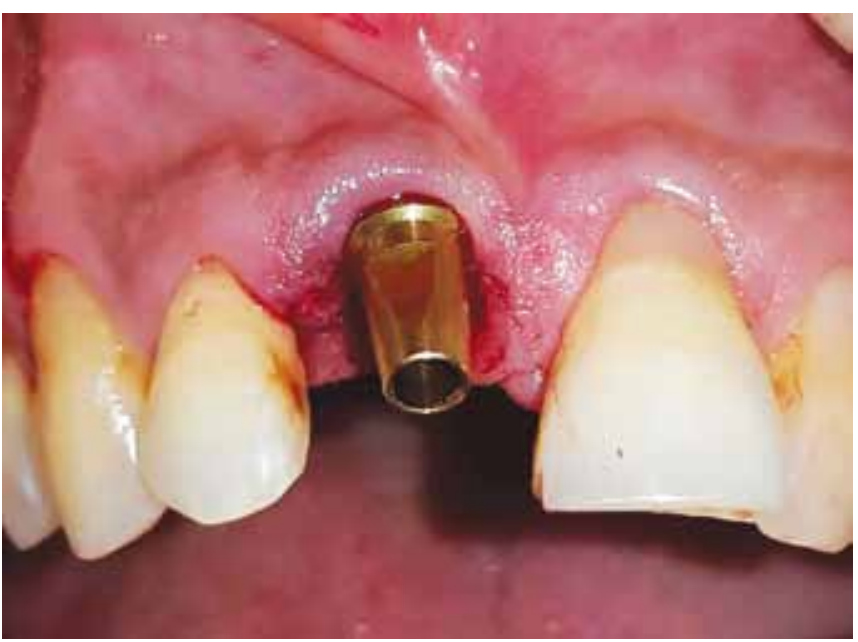

Fig. 11: Abutment placed

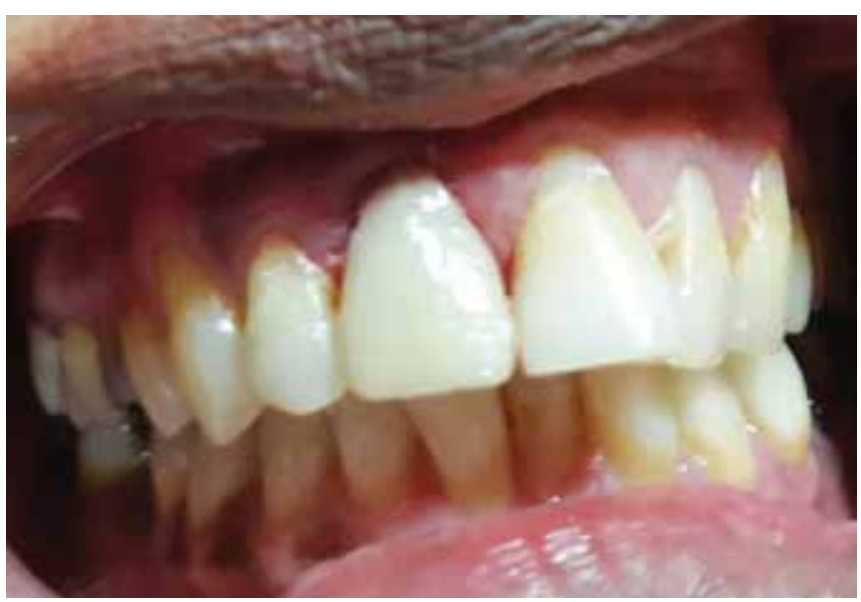

Fig. 13: Provisional restoration placed

Occlusal adjustments were made in the provisional restoration to ensure that it was free of protrusive and lateroprotrusive to promote osseointegration during the primary peri-implant bone remodeling phase.

After 120 days, the definitive restoration was initiated. At that moment, the gingiva was healthy and the concave arch and gingival papillae were not negatively affected. Stability of peri-implant soft tissues and of emergence profile was favored by the use of the crown of the natural tooth and by its cervical anatomy.

As the original gingival architecture was preserved, the transference of the emergence profile was made with vinyl polysiloxane impression material, and a final prosthesis was fabricated, it consisted of a full ceramic crown cemented on a customized, metal ceramic, UCLA abutment. The final margins of the crown were placed $1.5 \mathrm{~mm}$ below the gingival margin.

At the end of the clinical treatment, the patient received instructions about hygiene and the maintenance of the prosthesis. A follow-up program for visits every 6 months

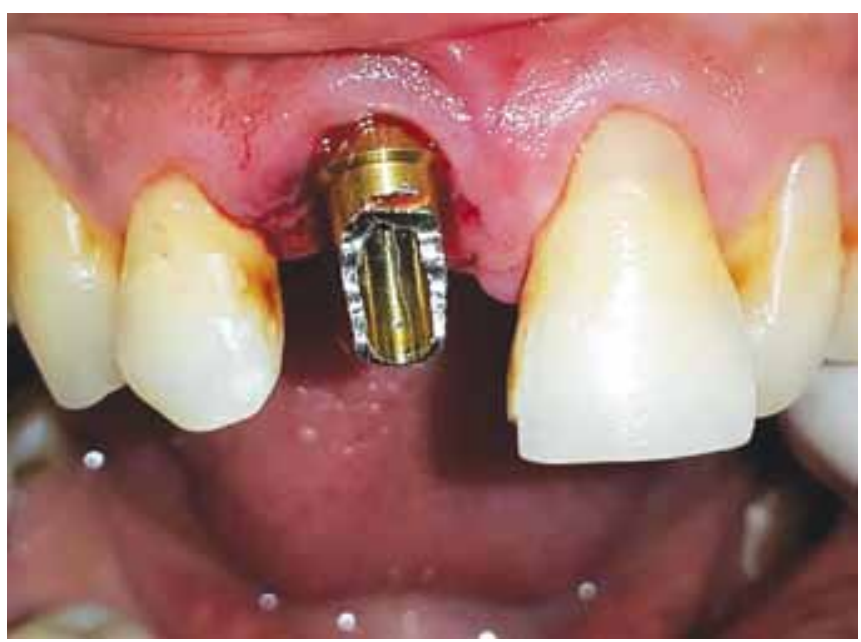

Fig. 12: Abutment milled to compensate for labial prominence

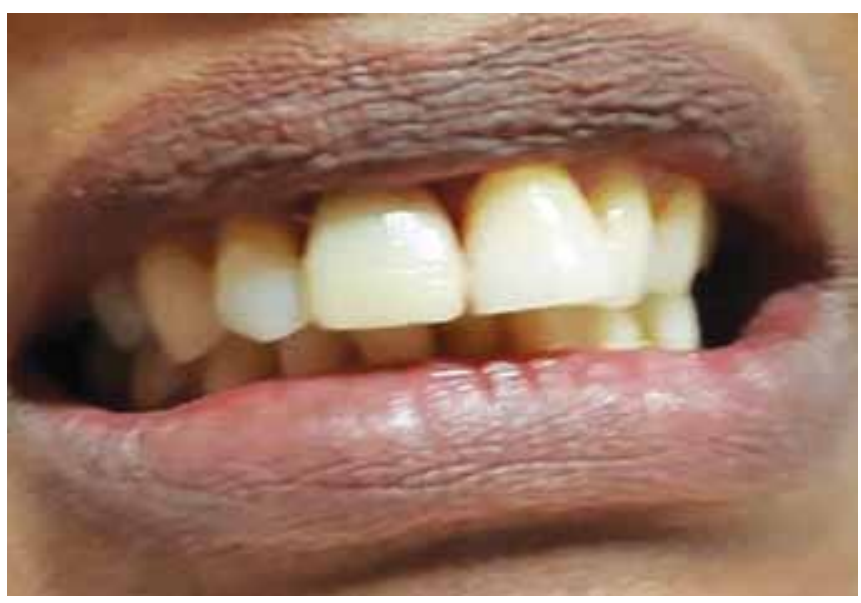

Fig. 14: Final postoperative view

was defined so that the level of satisfaction achieved could be maintained for the longest possible time.

The implant, abutment, and crown had been functioning for 12 months after surgery, and, to date, the esthetic outcome has been preserved; no new clinical or radiographic signs were observed, and no symptoms were reported by the patient.

\section{Case 2}

A 30 years old male came with the chief complaint of fractured upper left lateral incisor (tooth no. \#10) (Fig. 15), to the Department of Implantology, VS Dental College and Hospital, Bengaluru. Patient gave a history of root canal treatment done 3 months back, following which, patient underwent fixed dental prosthesis. On radiographic examination, a radiolucency signifying the fracture line was detected across the middle third of the root.

A treatment plan similar to the one described in the first case report was envisioned and executed to result in immediate implant placement following extraction, with 


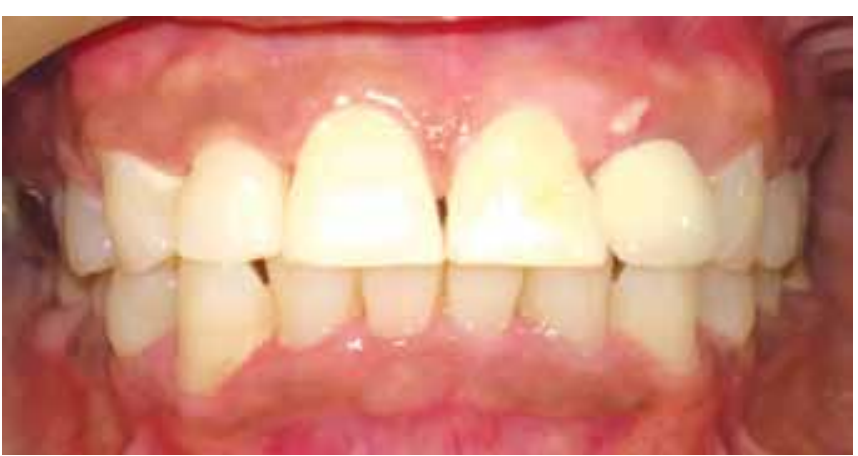

Fig. 15: Preoperative view

immediate loading, by creating a provisional restoration, using the crown portion of the extracted tooth (Fig. 16).

A satisfactory primary stability of $35 \mathrm{Ncm}^{2}$ was obtained, with good esthetics, satisfying the needs of the patient.

\section{Case 3}

A 35 years old female patient came with the chief complaint of fractured upper left lateral incisor (tooth no. \#10) (Fig. 17), to the Department of Implantology, VS Dental College and Hospital, Bengaluru. Patient gave a history of root canal treatment done 1 year back, following which, patient underwent fixed dental prosthesis. The tooth exhibited mobility following trauma. On radiographic examination, a radiolucency signifying the fracture line was detected across the middle third of the root.

A treatment plan similar to the one described in the first and second case reports was envisioned and executed to result in immediate implant placement following extraction, with immediate loading, by creating a provisional restoration (Fig. 18).

A satisfactory primary stability of $35 \mathrm{Ncm}^{2}$ was obtained, with good esthetics, satisfying the needs of the patient.

The summary of all the three cases has been presented (Table 1).

\section{DISCUSSION}

The outcome of these cases demonstrated that immediate implant placement is an excellent treatment option in areas with high esthetic demands, such as the anterior maxilla. The main advantages of this concept are the preservation of hard and soft tissues surrounding the implant, the reduced time for therapy completion and the immediate fixation of a transitional restoration.

However, not all cases can be treated following this protocol; in fact, certain prerequisites need to be met for a functional and esthetic result to be achieved.

The prognosis of root fractures, especially those involving the cervical and middle thirds of the root, depends not only on the length of the fracture line but also on the

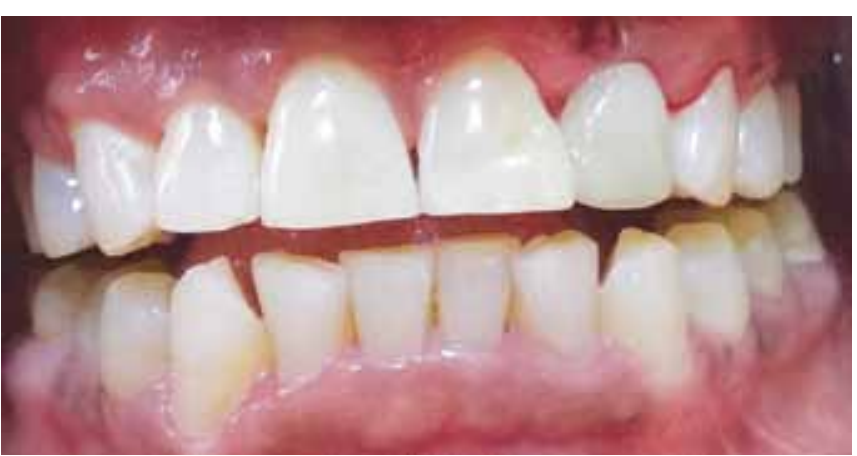

Fig. 16: Postoperative view

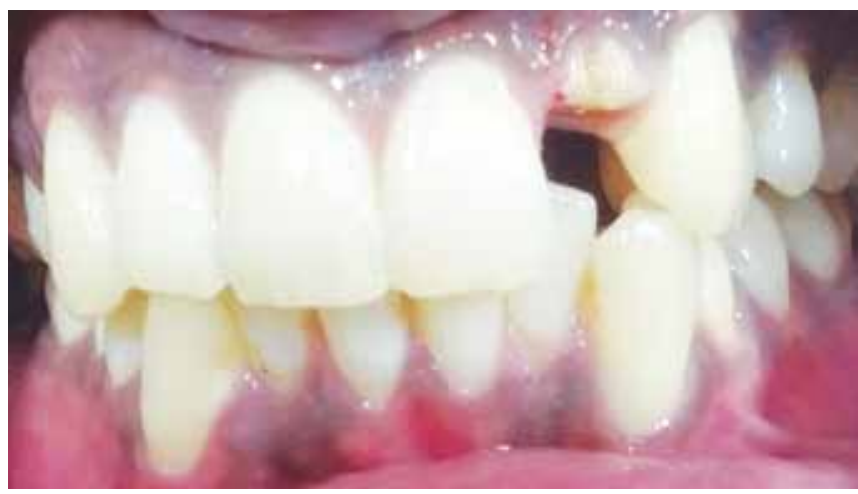

Fig. 17: Preoperative view

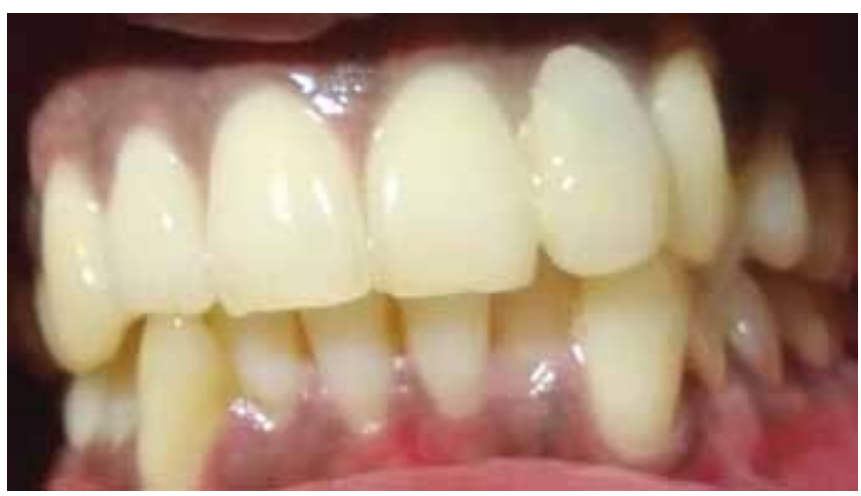

Fig. 18: Postoperative view

Table 1: Summary of the cases presented under this case series

\begin{tabular}{lllll}
\hline Tooth & Patient & & $\begin{array}{l}\text { Immediate } \\
\text { Immediate } \\
\text { implant } \\
\text { number }\end{array}$ & $\begin{array}{l}\text { lization } \\
\text { without } \\
\text { functional }\end{array}$ \\
\hline$\# 8$ & placement & loading \\
$\# 10$ & 30/F & Yes & Yes & Yes \\
$\# 10$ & Y5/F & Yes & Yes & Yes \\
\hline
\end{tabular}

condition of the pulp and occlusion, on the presence or absence of root fragment displacement, and on general and oral health of the patient. At the same time, the prognosis should include adequate treatment plan according to clinical and radiologic findings. ${ }^{5}$ In the cases reported here, the 
patients had no systemic disorders and, due to trauma, had a fracture at the junction of the middle and cervical third of the root of the maxillary anteriors.

The prognosis of cervical root fractures depends on the type and site of the fracture line. Their prognosis is better than those that follow the long axis of the root. At the same time, transverse cervical fractures have a worse prognosis than oblique fractures, probably because of the possible micromovements that may occur after treatment and which may lead to further luxation. Such displacements may be caused by even minor impact generated by mastication or occlusion itself. ${ }^{6}$ Statistically, maxillary central incisors are the teeth most often affected (75\%) in cases of dental trauma, particularly due to their natural projection in the anterior maxilla, which makes them more susceptible to structural injury. Maxillary lateral incisors are also affected by a high percentage of trauma and account for $21 \%$ of the cases. ${ }^{6}$

Several techniques are available for the restoration of a lost tooth, such as the use of fixed or removable prosthesis and, currently, tooth replacement using osseointegrated implants. ${ }^{7}$

Socket wall integrity, especially at the labial aspect, is ensured with the use of a periodontal probe; because this technique is blind, the possible existence of fenestrations, uneven crestal resorption, or trauma could jeopardize implant survival. In this respect, the extraction has to be atraumatic. The use of the periotome facilitates the procedure by enlarging the space between root and socket walls and, thus, eliminating the need for conventional elevators.

Conventional instruments could impinge on the papillae and cause damage to this area. If the root appears dilacerated and the extraction is anticipated as difficult or complicated, a more conventional approach might be preferable.

In the presented cases, the soft-tissue trauma was minimal and no further signs of acute or chronic infection were traced. Although preexisting periodontal/disease or periapical pathosis in animal studies did not impair bone remodeling around implants if properly treated, ${ }^{8,9}$ we, among others think it is prudent to avoid immediate implant placement into infected sockets because of the potential risk of future implant periapical lesion development. ${ }^{10,11}$

The implants selected were wide-diameter root form instead of a cylindrical ones. This shape allowed for almost complete filling of the socket by the fixture and limited the peri-implant space to less than $2 \mathrm{~mm}$ in the coronal part. Under these clinical conditions, the use of grafts and/or membranes was not necessary. ${ }^{12}$

To increase chances of success and to allow the use of immediate loading with a provisional, initial implant locking pressure should be high, about $35 \mathrm{Ncm}^{2}$
Therefore, the measurement of insertion torque at the time of implant placement should determine whether the prosthesis can be placed and the provisional crown fabricated immediately. Also, micromovements generated by occlusion and mastication should be minimized to avoid intercuspation and eccentric contacts, and the provisional prosthesis should not be removed during the initial phases of peri-implant bone repair. ${ }^{13,14}$

The surgery protocol should include the evaluation of bone quality and quantity as well as measures to ensure that the placement of the prosthesis and other components is adequate. ${ }^{15}$ In the cases described here, in addition to an initial locking pressure greater than $35 \mathrm{Ncm}^{2}{ }^{2}$ careful provisional fabrication and adjustments ensured that the esthetic results were good and avoided centric and eccentric contacts.

A critical factor in implant rehabilitation of teeth in the anterior maxilla is the three-dimensional positioning of implants. Implant insertion at a palatal position in relation to the alveolar ridge (palatal approach) preserves the buccal bone wall and provides better implant locking. Therefore, it reduces the incidence of future gingival recession and implant exposure. ${ }^{16}$

A cohort study found that the use of a palatal approach and bone autografts in the socket had successful results in $94 \%$ of the cases analyzed. In our case, the use of the palatal approach preserved the buccal wall of the socket, which was filled only with the clot, and kept in place by the edge of the provisional prosthesis itself. ${ }^{17}$

One of the advantages of immediate loading is the restoration of function and esthetics immediately after surgery. Moreover, the adjacent gingival papillae are preserved, and a second surgery is not necessary. ${ }^{11}$

Also, the use of a removable provisional prosthesis for a long time while waiting for rehabilitation with a permanent prosthesis may be a problem, but not when the technique described here is used, because the provisional prosthesis is inserted and fixed immediately after surgery. In the clinical case described here, there was an important psychological gain as the use of a partial removable prosthesis was avoided.

Screw-retained provisional crowns may be used in the anterior and posterior maxilla, but cemented crowns are preferred for the anterior maxilla due to the inclination of the premaxilla and the fact that the retention screw may transverse the buccal surface of the tooth. ${ }^{17}$ Therefore, we chose implant temporization using the provisional, fabricated chairside, cemented on the abutment.

There was an important esthetic gain because the natural gingival contour was preserved. Moreover, the provisional crown served as a model to fabricate the definitive crown after osseointegration was consolidated. 
These clinical cases showed that, using the acrylic tooth which modifies chairside to fabricate, provisional prosthesis is one of the several possible alternatives for temporization.

The preservation of esthetics as well as of the charac teristics of adjacent teeth and of gingival harmony produced good results, facilitated fabrication of the definitive prosthesis and did not affect the individual characteristics of the patient's smile.

\section{CONCLUSION}

Tooth extraction, immediate implant placement and provisionalization with immediate loading, is a treatment option which offers several advantages compared to the conventional two-stage protocols, such as simplicity, superior esthetic outcome, immediate fixed restoration and reduced time required for the final restoration. However, more research in this field and long-term clinical studies are necessary to corroborate the results of this report.

\section{REFERENCES}

1. Lazzara R. Immediate implant placement into extraction sites: surgical and restorative advantages. Int J Periodontics Restorative Dent 1989;9(5):333-343.

2. Barzilay I, Graser GN, Iranpur B, et al. Immediate implantation of a pure titanium implant into an extraction socket: report of pilot procedure. Int J Oral Maxillofac Implants 1991;6(3): 277-284.

3. Tolman DE, Keller EE. Endosseous implant placement immediately following dental extractions and alveoplasty: preliminary report with 6-year follow-up. Int J Oral Maxillofac Implants 1991;6(1):24-28.

4. Gelb DA. Immediate implant surgery: three-year retrospective evaluation of 50 consecutive cases. Int J Oral Maxillofac Implants 1993;8(4):388-399.

5. Schwartz-Arad D, Chaushu G. Placement of implants into fresh extraction sites: 4 to 7 years retrospective evaluation of 95 immediate implants. J Periodontol 1997;68(11):1110-1116.
6. Gomez-Roman G, Kruppenbacher M, Weber H, Schulte W. Immediate postextraction implant placement with root analog stepped implants: surgical procedure and statistical outcome after years. Int J Oral Maxillofac Implants 2001;16(4):503-513.

7. Locante WM. The nonfunctional immediate provisional in immediate extraction sites: a technique to maximize esthetics. Implant Dent 2001;10(4):254-258.

8. Vergara JA, Caffesse RG. Preservation of esthetics with implant dentistry: a clinical report. Clin Implant Dent Relat Res 2002;4(4):200-211.

9. Schiroli G. Immediate tooth extraction, placement of a tapered screw-vent implant, and provisionalization in the esthetic zone: a case report. Implant Dent 2003;12(2):123-131.

10. Norton MR. A short-term clinical evaluation of immediate TiOblast single-tooth implants. Int J Oral Maxillofac Implants 2004;19(2):274-281.

11. Nuzolesse E. Immediate loading of two single tooth implants in the maxilla: preliminary results after one year. J Contemp Dent Pract 2005;6(3):148-157.

12. Novaes AB Jr, Vidigal GM Jr, Novaes AB, Grisi MF, Polloni S, Rosa A. Immediate implants placed into infected sites: a histomorphometric study in dogs. Int J Oral Maxillofac Implants. 1998;13(3):422-427.

13. Marcaccini AM, Novaes AB Jr, Souza SL, Taba M Jr, Grisi MF. Immediate placement of implants into periodontally infected sites dogs. Part 2: a fluorescence microscopy study. Int J Oral Maxillofac Implants 2003;18(6):812-819.

14. Jalbout ZN, Tarnow DP. The implant periapical lesion: four reports and review of the literature. Pract Proced Aesthet Dent 2001;13(2):107-112.

15. Tsoukalas D, Karamanis S, Rallis M, Parissis N. Implant periapical lesion: case report and literature review. Balk J Stom 2004;8:154-158.

16. Nyman S, Lang NP, Buser D, Braggen U. Bone regeneration adjacent to titanium dental implants using guided tissue regeneration: a report of two cases. Int J Oral Maxillofac Implants 1990;5(1):9-15

17. Becker W, Becker BE. Guided tissue regeneration for implants placed into extraction sockets and for implant dehiscencsurgical techniques and case reports. Int J Periodont Restor Dent 1990;10(5):376-391. 